\title{
CIANOSE E PÚRPURA NOS QUIRODÁCTILOS - FUNDAMENTOS DO DIAGNÓSTICO DIFERENCIAL, A PROPÓSITO DE UM CASO
}

\author{
Fred Bernardes Filho', Bianca Passos Leite dos Santos², Mariorie Soares Pereira Gonçalves³, Suzana Mary de Carvalho4, \\ Viviane Vargas dos Santos ${ }^{5}$ \\ 'Pós Graduando de Dermatologia/Graduated in Dermatology, Instituto de Dermatologia Professor Rubem David Azulay, \\ Santa Casa da Misericórdia do Rio de Janeiro (IDPRDA/SCMRJ), Brasil \\ ${ }^{2}$ Universidade Estacio de Sa/Estacio de Sa University, Rio de Janeiro, Brasil \\ ${ }^{3}$ Universidade Fluminense/Fluminense Federal University, (UFF), Niterói (RJ), Brasil \\ ${ }^{4}$ Universidade Federal do Rio de Janeiro/Federal University of Rio de Janeiro (UFRJ), Brasil \\ ${ }^{5}$ Departamento de Medicina Interna/Department of Internal Medicine, Hospital de Caridade de ljuí, Rio Grande Federal \\ University, (HCI/FURG), Rio Grande (RS), Brasil.
}

\begin{abstract}
RESUMO - As púrpuras e a cianose são lesões elementares frequentemente vistas por médicos de diversas especialidades. Suas causas podem variar de doenças raras, como trombofilias, a traumatismos ocupacionais. Os fundamentos da formação destas lesões devem ser de domínio dos dermatologistas para que possam auxiliar no diagnóstico correto da doença de base. Os autores relatam o caso de um paciente em internação hospitalar que apresentava cianose e equimose nos dígitos causadas por traumatismos repetitivos nos dedos para a aferição da glicemia capilar. Os possíveis diagnósticos diferenciais do caso em questão são revisados.
\end{abstract}

PALAVRAS-CHAVE - Cianose; Dedos; Púrpura.

\section{CYANOSIS AND PURPURA IN FINGERS - FUNDAMENTALS OF THE DIFFERENTIAL DIAGNOSIS, APROPOS OF A CASE}

ABSTRACT - The purpura and cyanosis are elementary lesions frequently seen by medical doctors of several specialties. Its causes can range from rare diseases, such as thrombophilia, to the occupational injuries. The fundamentals of the formation of these lesions should be domain of dermatologists so that they can aid in the correct diagnosis of the underlying disease. The authors report the case of a patient in hospital who had cyanosis and ecchymosis in the digits caused by repetitive trauma to fingers in the measurement of capillary blood glucose. The differential diagnosis of the case is reviewed.

KEY-WORDS - Cyanosis; Fingers; Purpura.

Conflitos de interesse: Os autores declaram não possuir conflitos de interesse.

No conflicts of interest.

Suporte financeiro: $O$ presente trabalho não foi suportado por nenhum subsídio ou bolsa.

No sponsorship or scholarship granted.

Direito à privacidade e consentimento escrito / Privacy policy and informed consent: Os autores declaram que pediram consentimento ao doente para usar as imagens no artigo. The authors declare that the patient gave written informed consent for the use of its photos in this article.

Recebido/Received - Agosto/August 2014; Aceite/Accepted - Setembro/September 2014 


\title{
Caso Clínico
}

\author{
Correspondêncio: \\ Dr. Fred Bernardes Filho \\ Marquês de Caxias, $n^{\circ}$ 9, Sobrado, Centro \\ 24030-050. Niterói (RJ), Brasil \\ Tel: +55 (21) 98092-9535 \\ Fax: +55 (21) 3619-1511 \\ Email: f9filho@gmail.com
}

\section{INTRODUÇÃO}

A semiologia define cianose como coloração azul-arroxeada da pele. Ocorre por aumento da hemoglobina reduzida no sangue capilar além de $5 \mathrm{~g} \%$ (o normal é em torno de $2,6 \mathrm{~g} \%)$. A cianose não é detectável até que a saturação de oxigênio no sangue seja menor que $85 \%{ }^{1}$. Quanto à lesão purpúrica, ela decorre de uma pequena hemorragia dos vasos da derme e/ou hipoderme, cujas hemácias extravasadas são fagocitadas pelos macrófagos que transformam o pigmento hemoglobínico em hemossiderina.

Há três tipos de cianose. Cianose central: resultado de hipoxemia arterial; cianose periférica: associada à estase, a qual permite uma extração mais acentuada de oxigênio da hemoglobina contida no sangue periférico; cianose por alteração da hemoglobina (Tabela 1) ) $^{3,4}$.

Os autores descrevem o caso de um paciente com pneumonia que durante a internação hospitalar apresentou cianose e petéquias nos dígitos. Após a exclusão de outras doenças causadoras de cianose e avaliação cuidadosa das lesões, concluiu-se tratar-se de cianose mista. Os traumatismos repetidos nos quirodáctilos para a aferição da glicemia capilar, resultando, inclusive nas petéquias observadas, associado ao quadro de pneumonia, resultaram na cianose digital apresentada.

\section{CASO CLÍNICO}

Paciente do sexo masculino, 71 anos de idade, hipertenso e diabético, foi internado por quadro de pneumonia e fibrilação atrial de alta resposta. À admissão hospitalar foi verificado descontrole glicêmico e indicada substituição dos hipoglicemiantes orais por insulina. Durante a internação, houve piora do padrão respiratório com aumento da extensão da pneumonia. A partir do quinto dia de hospitalização, surgiram lesões purpúricas e cianose nos quirodáctilos.

Os exames laboratoriais demonstravam leucocitose $\left(18.000\right.$ leucócitos $\left./ \mathrm{mm}^{3}\right)$ com neutrofilia acentuada (90\% de neutrófilos); eletrólitos e bioquímica não eletrolítica dentro dos padrões de normalidade. Em seguida, foi solicitado parecer da dermatologia pela equipe de clínica médica.

Ao exame dermatológico, as falanges distais dos quirodáctilos apresentavam um aspecto cianótico (Fig. 1). Foram observadas equimoses, petéquias, múltiplas lesões perfurantes e cianose nas polpas digitais dos dedos

Tabela 1 - Tipos de cianose.

TIPO

Cianose central

Cianose periférica
Cianose por alteração da hemoglobina
- Exposição ao frio (vasoconstrição)- mais comum

- Insuficiência congestiva grave (congestão periférica)

- Doença de Raynaud (transtorno vasomotor)

- Acrocianose (transtorno vasomotor com espasmos de pequenos capilares ou artérias)

\section{CAUSAS}

- Redução da concentração de oxigênio (altitudes elevadas)

- Alteração da ventilação pulmonar (tumores, enfisema pulmonar, atelectasia)

- Transtornos de difusão (infecções, fibrose pulmonar, edema de pulmão na insuficiência cardíaca)

- Transtornos de perfusão (insuficiência cardíaca grave, embolia pulmonar, cardiopatias congênitas)

- Shunts arteriovenosos (Tetralogia de Fallot, tronco arterioso, Síndrome de Eisenmenger, atresia tricúspide, comunicação interatrial ou interventricular com hipertensão pulmonar e fístulas vasculares pulmonares)

Metemoglobinemia 


\section{Caso Clínico}

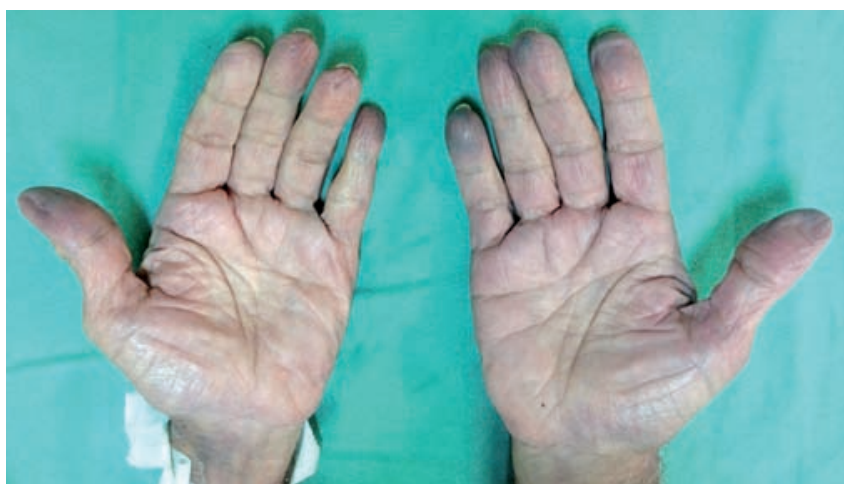

Fig 1 - Aspecto cianótico das falanges distais dos quirodáctilos.

das mãos (Fig.s 2 e 3). Apesar da dieta apropriada e uso da insulina suplementar para controle da hiperglicemia, foi difícil obter o controle glicêmico do paciente. Em virtude da necessidade do rígido controle da glicemia, foram realizadas avaliações da glicemia capilar a cada 3 horas e os múltiplos ferimentos provocados pela lanceta nas polpas digitais resultaram em equimoses e petéquias das falanges distais, simulando um quadro de isquemia periférica. Tais traumatismos múltiplos ainda resultaram em lesão endotelial, aumento da permeabilidade capilar com prejuízo na microcirculação resultando em cianose periférica e simulação de quadro de isquemia periférica. Além disso, o quadro de pneumonia extensa favoreceu o surgimento de uma cianose central por distúrbio na difusão e ventilação pulmonar.

Assim, concluímos tratar-se de cianose mista, associada a petéquias e equimoses. A evolução foi boa, havendo desaparecimento das lesões após a melhora cínica e a interrupção da realização de múltiplas puncturas.

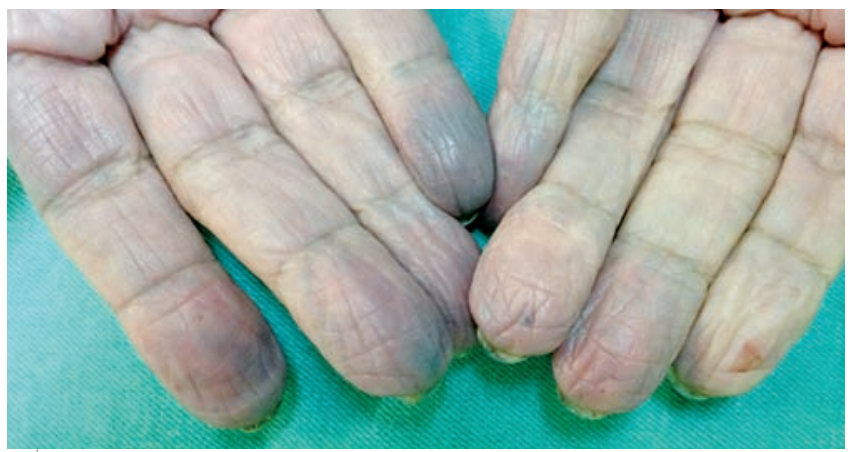

Fig 2 - Lesões equimóticas nas polpas digitais dos quirodáctilos e presença de múltiplas lesões perfurantes provocadas para realização da glicemia capilar.

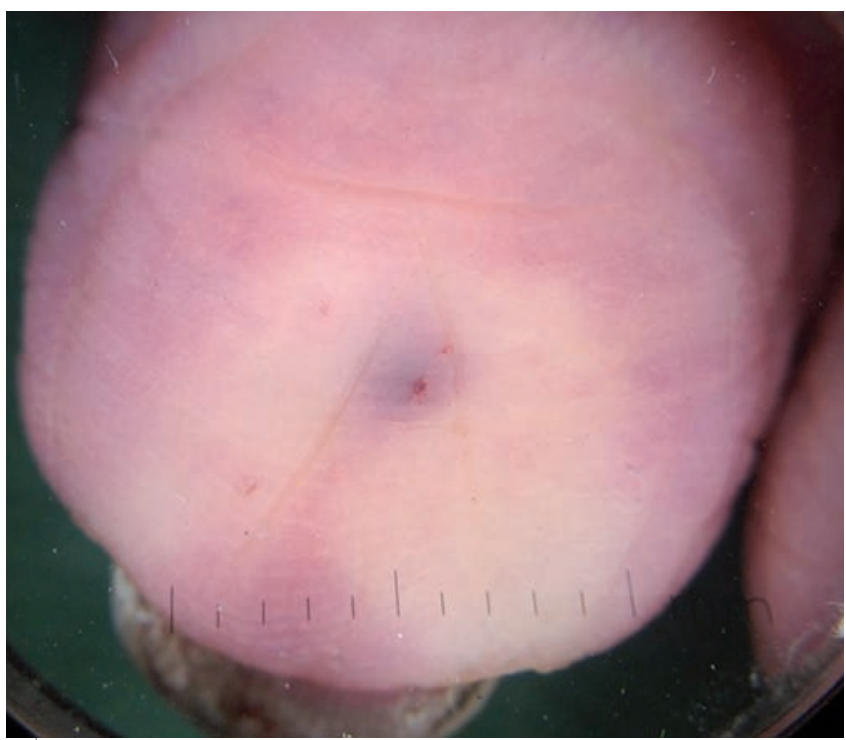

Fig 3 - Detalhe do local da punctura para realização da glicemia capilar, com equimose perilesional. A área anêmica da falange é resultado da vitropressão provocada pelo uso do dermatoscópico.

\section{DISCUSSÃO}

As lesões purpúricas são classificadas em petéquia (lesão puntiforme) e equimose (lesão purpúrica em lençol). O hematoma é uma coleção maior, em geral, de natureza traumática ${ }^{2}$. As lesões purpúricas diferem das lesões eritematosas porque não desaparecem à vitropressão ou digitopressão ${ }^{2,5}$. A coloração da lesão, em alguns casos, pode fornecer pistas do tempo de sua evolução. O sangue extravasado na pele será reabsorvido, e isso provoca variação cromática desde o início ao pleno reparo da lesão. Essa mudança de tons é conhecida como espectro equimótico de Legrand du Saulle e tem importante valor na traumatologia forense. Algumas regiões, por peculiaridades anatômicas, não apresentam o espectro equimótico. São elas a conjuntiva ocular, os dedos e as regiões palmo-plantares ${ }^{5,6}$. As púrpuras podem ser divididas em dois grupos: trombocitopênicas (contagem baixa de plaquetas) e não trombocitopênicas (contagem normal de plaquetas). Na etiopatogenia das púrpuras que ocorrem nas extremidades, devem-se pesquisar drogas, infecções (endocardite infecciosa, meningococcemia) e outras doenças de base ${ }^{1,2,5}$. Traumatismos ocasionais e ocupacionais como repetidas puncturas feitas por costureiras e bordadeiras também podem produzir petéquias e equimoses nos dígitos, semelhante ao caso apresentado. 


\section{Caso Clínico}

Merece destaque o fato de que algumas dermatoses são observadas com maior frequência nos pacientes diabéticos, tais como candidíase cutânea, dermatofitoses disseminadas ou recorrentes, acantose nigricans, mal perfurante plantar, entre outras. Em alguns casos, pode-se observar atrofia decorrente da própria aplicação da insulina ${ }^{7,8}$.

Embora os hipoglicemiantes orais sejam amplamente utilizados em pacientes ambulatoriais, existem muitas desvantagens em mantê-los durante a internação. Os agentes orais só devem ser mantidos no paciente com diabetes mellitus tipo 2, bem controlado, em internações eletivas, de curta duração, desde que não concorram com outros medicamentos ou com alterações nutricionais que possam deteriorar o controle glicêmico?. Ao contrário destes, a insulina age rapidamente, responde bem às titulações da dose, e pode ser utilizada em praticamente todos os pacientes, e em qualquer condição clínica para controlar a glicemia. Durante a internação é preconizada a avaliação da glicemia capilar quatro vezes por dia (pré-refeições e às 23 horas), sendo esta realizada através de uma picada superficial com uma lanceta ou agulha na ponta do quirodáctilo do paciente? Nos casos de internação prolongada, lesões similares a estas apresentadas no caso em questão podem ocorrer. Estes sinais clínicos, cianose e púrpuras, se somados aos sintomas podem direcionar ao correto diagnóstico.

Os autores concluem que os fundamentos da formação das púrpuras e cianose e os diversos diagnósticos diferenciais que podem apresentar estas lesões elementares devem ser de domínio dos dermatologistas. O correto diagnóstico da doença de base exige um raciocínio semiológico necessário ao médico assistente quando se vê frente a estes tipos de lesões, no dia-a-dia ambulatorial ou em interconsultas de especialidades nas enfermarias hospitalares. Ressalta-se que a dermatoscopia ganha a cada dia mais importância na prática clínica, com o aumento do número de suas indicações, acrescentando nova dimensão ao exame clínico. Os autores destacam o dermatoscópio como um valioso instrumento no correto diagnóstico do caso apresentado.

\section{REFERÊNCIAS}

1. Porto CC. Semiologia Médica. $4^{\text {th }}$ ed. Rio de Janeiro: Guanabara Koogan; 2001.

2. Krause K, Azulay RD. Púrpuras. In Azulay RD, Azulay DR, Azulay-Abulafia L, eds. Azulay Dermatologia. Rio de Janeiro: Guanabara Koogan; 2013. p. 344-6.

3. Rashid BA, Houshmand EB, Heffernan MP. Hematologic Diseases. In Fitzpatrick TB, Eisen AZ, Wolff $\mathrm{K}$, Freedberg IM, Austen KF, eds. Dermatology in general medicine. $7^{\text {th }}$ ed. New York: McGraw-Hill; 2008. p. 1373-1385.

4. Ferreira JC, Carvalho CRR. Insuficiência Respiratória Aguda. In Lopes AC, editor. Tratado de Clínica Médica. São Paulo: Roca; 2006. p. 46454649.

5. Cox NH, Coulson IH. Diagnosis of Skin Disease. In Burns T, Breathnach S, Cox N, Griffiths C, eds. Rook's Textbook of Dermatology. 8h $^{\text {th }}$ ed. Oxford: Wiley Blackwell; 2010. p. 5.1-25.

6. Bernardes Filho F, Quaresma MV, Paolini KS, Rocha NC, Kac BK, Azulay-Abulafia L. Talon noir: dermoscopy assisted differential diagnosis of pigmented lesions. Surg Cosmet Dermatol 2013; 5:151-3.

7. Behm B, Schreml S, Landthaler M, Babilas P. Skin signs in diabetes mellitus. J Eur Acad Dermatol Venereol. 2012; 26:1203-11.

8. Roselino AMF. Doenças de origem metabólica e nutricional. In Ramos-e-Silva M, Castro MCR eds. Fundamentos de Dermatologia. Rio de Janeiro: Atheneu; 2010:1097-114.

9. Diabetes.org.br [página na internet]. Controle da hiperglicemia intra-hospitalar em pacientes críticos e não críticos [acesso 8 Abr 2014]. Disponível em: http://www.diabetes.org.br/attachments/posicionamento/posicionamento-sbd-n-02-2011. pdf 